\title{
Distribution of JC Virus DNA in Peripheral Blood Lymphocytes of Hematological Disease Cases
}

\author{
Naoko Shimizu, Akira Imamura, Osami Daimaru, Hidetsugu Mihara, Yoshiro Kato, Ryoichi Kato, \\ Takashi Oguri, Masako FukadA*, Takashi Yokochi*, Kazuhiro YoshikawA**, Hirokazu Komatsu***, \\ Ryuzo UedA*** and Masakazu NiTTA
}

\begin{abstract}
Objective The distribution of JC virus DNA in peripheral blood was surveyed by the polymerase chain reaction using the late genes as markers. Results Six out of 52 cases of hematological diseases and one systemic lupus erythematosus case out of 17 cases were positive for JCV DNA. After separation into B and T lymphocytes by a cell sorter, JCV DNA was found in both cell types prepared from adult $T$ cell leukemia and PML patients. Conclusion Only 1 or 2 copies of JCV genome were calculated to exist in a cell based on the time course analysis of PCR. Only B lymphocytes and glial brain cells are known to produce nuclear factors which support the growth of the virus. The result that B lymphocytes contained a copy number of JCV genome similar to T lymphocytes suggests that there is some barrier to viral growth in susceptible B lymphocytes, and that the growth of $\mathrm{JCV}$ is different from that of other virulent viruses.
\end{abstract}

(Internal Medicine 38: 932-937, 1999)

Key words: human polyoma virus, progressive multifocal leukoencephalophathy, agno gene, VP-1 gene, hematological malignancy

\section{Introduction}

Progressive multifocal leukoencephalophathy (PML) was originally described as extensive demyelination associated with chronic lymphocytic leukemia (CLL) and Hodgkin's lymphoma (1). The JC virus (JCV), a human polyoma virus isolated from the brain of a PML patient (2), infects early in life and persists in the kidney under the latent state. Over $70 \%$ of adults contain the antibody to JCV (3). With the progression of immunosuppressive states (aging, presence of malignant tumors, or hematological diseases), JCV is reactivated and presumably migrates via B lymphocytes into the brain, where it causes PML (3).

During the shift from latency to onset of PML, JCV may be distributed not only in B lymphocytes, kidney and brain, but in other cells and organs as well. The distribution of JCV was previously surveyed in brain, cerebro-spinal fluid (CSF) and urine by polymerase chain reaction (PCR) (4). As a typical step in immunodeficiency, patients with hematological diseases, hematological malignancies, or autoimmune diseases were adopted as the source of peripheral blood. The early genes code for proteins mostly controlling and regulating viral multiplica- tion. On the other hand, the late genes code for viral structural proteins, and are better indexes for viral particles (5). Using newly established primers for agno gene and VP-1 gene, we searched JCV DNA by PCR in peripheral blood of clinical patients. The positive control was a brain specimen from a PML case autopsy and peripheral lymphocytes of 20 healthy volunteers were applied as the negative control.

\section{Materials and Methods}

\section{Cells}

Peripheral lymphocytes: cells from 1 PML patient, 2 adult T cell leukemia (ATL), 2 acute lymphoblastic leukemia (ALL), 5 acute myeloblastic leukemia (AML), 7 CLL, 2 chronic myelogenous leukemia (CML), 4 myelodysplastic syndrome (MDS), 28 malignant lymphoma (ML), 2 multiple myeloma, and 17 systemic lupus erythematosus (SLE) patients were examined.

\section{Flow cytometry}

Peripheral blood was collected in heparinized tubes from

From the Second Department of Internal Medicine, Aichi Medical University, Nagakute, *Department of Microbiology and Immunology, Aichi Medical University, **the Second Department of Pathology, Aichi Medical University and ***the Second Department of Internal Medicine, Nagoya City University Medical School, Mizuho

Received for publication September 24, 1998; Accepted for publication June 23, 1999

Reprint requests should be addressed to Dr. Naoko Shimizu, the Second Department of Internal Medicine, Aichi Medical University, Nagakute, Aichi-gun, Aichi 480-1195 
patients after obtaining informed consent. Lymphocytes were isolated from each sample by centrifugation on Lymphoprep (NYCOMED PHARMA AS, Oslo) at $400 \times \mathrm{g}$ for 20 minutes. The cell number was adjusted to $10^{6} / \mathrm{ml}$ in PBS and incubated for 30 minutes on ice with $200 \mu \mathrm{l}$ of anti-CD3/anti-CD19 dual antibodies (DACO A/S, Denmark). After sorting, the resulting $\mathrm{T}$ and $\mathrm{B}$ lymphocytes were re-analyzed on a flow cytometer to assess purity. The purity of both $\mathrm{T}$ and $\mathrm{B}$ lymphocytes was $95 \%$.

\section{DNA extraction}

Before DNA extraction, the cell number of all samples was adjusted to $10^{6} / \mathrm{ml}$. DNA was extracted using QIA amp Blood Kit (QIAGEN K. K. Japan).

\section{PCR}

The PCR solution consisted of $1 \times$ PCR buffer, $1.5 \mathrm{mM}$ $\mathrm{MgCl}_{2}, 200 \mu \mathrm{M}$ of each d-NTP, $1.0 \mu \mathrm{M}$ of each primer, 2.5 units of Ampli Taq Gold (Perkin Elmer, Cetus, USA), and 0.01 ng of JCV DNA (Mad-1 strain, prototype JCV) or DNAs precipitated from $2.5 \times 10^{4}$ cells. Sterile water was added to bring the volume to $100 \mu \mathrm{l}$ (5). Primers were agno \#1 (5'TTCGCCAGCTGTCACGTAA-3') (284 302) and agno \#2 (5'ATGTAGCTTTTGGTTCAGG-3') (490 472) for the agno gene, and VP-1 \#1 (5'-AAGTCAATATCTATATCAGATACA$\left.3^{\prime}\right)$ (1646 1669) and VP-1 \#2 (5'-AGTTGCTTGCCCAT TAGAG-3') (1852 1834) for the VP-1 gene (4). The PCR round consisted of 35 cycles of amplification for 1 minute at $94^{\circ} \mathrm{C}, 2$ minutes at $62^{\circ} \mathrm{C}, 1$ minute at $72^{\circ} \mathrm{C}$ with agno primers, 1 minute at $94^{\circ} \mathrm{C}, 2$ minutes at $55^{\circ} \mathrm{C}$, and 1 minute at $72^{\circ} \mathrm{C}$ with VP-1 primers. A 10 -minutes denaturing step at $94^{\circ} \mathrm{C}$ before the first cycle and a final elongation step of 10 minutes at $72^{\circ} \mathrm{C}$ in the last cycle were included.

\section{Detection of $P C R$ products}

After each PCR procedure, amplification products $(10 \mu \mathrm{l})$ were electrophoresed on a $3 \%$ agarose gel and visualized under UV light after soaking the gel in $0.5 \mu \mathrm{g} / \mathrm{ml}$ of ethidium bromide. The expected size was $207 \mathrm{bp}$ for the agno gene (base number of 284 to 490 ) and $207 \mathrm{bp}$ for a part of VP-1 gene (base number of 1646 to 1852) (Fig. 1).

\section{Results}

\section{Verification of PCR products}

As a positive control, DNA was extracted from peripheral lymphocytes of a PML patient and amplified by PCR using agno primers and VP-1 primers. Lanes B, C, and D in Fig. 2 showed a single band of the expected size ( $207 \mathrm{bp}$ for JCV agno) which corresponded to the one (lane A) of Mad-1 (prototype JCV) (Fig. 2). A similar result was obtained for VP-1 primers (Fig. 2; lane a d). Figure 3 shows that DNA isolated from peripheral lymphocytes of hematological malignancy contained a part of VP-1 genome (lane b and c), just as Mad-1 DNA did (lane a). As shown in Fig. 1, the amplified region of VP-1 genome is digested into two fragments, $77 \mathrm{bp}$ and 130

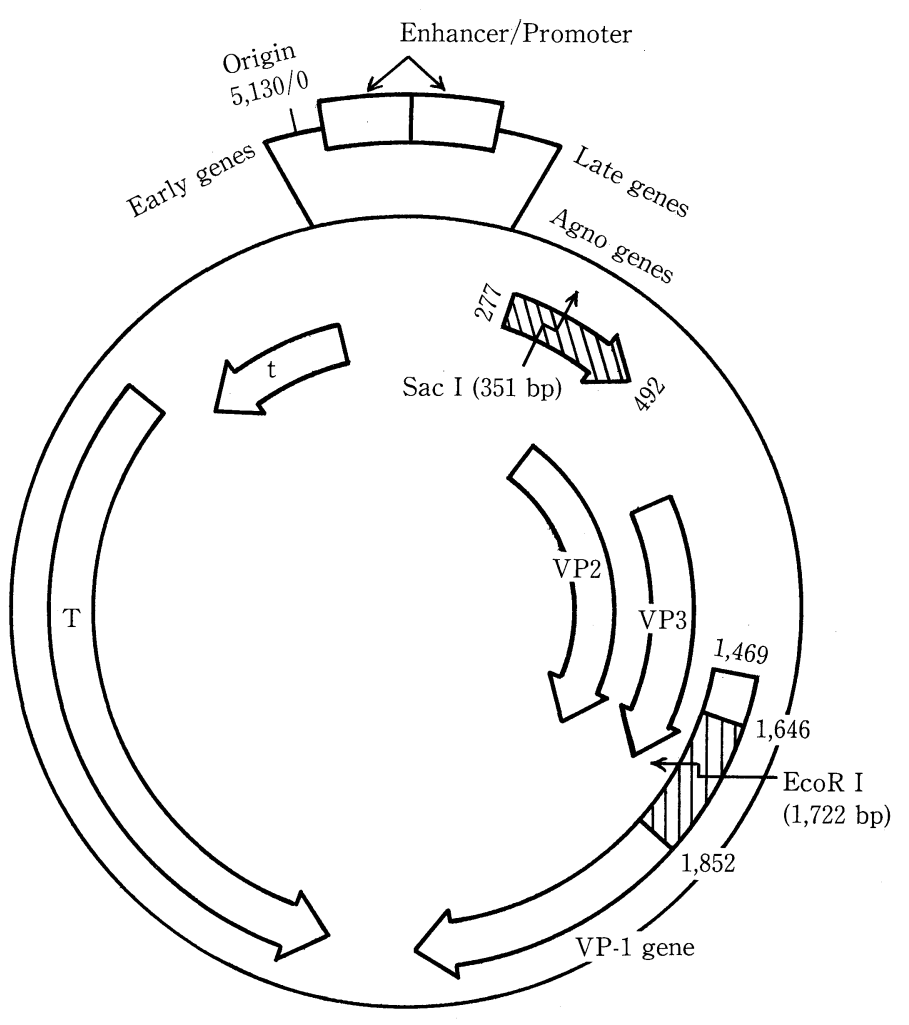

Figure 1. Genetic map of JCV. The origin of replication is taken as map position 0 on JCV genome, and bases are numbered clockwise. Agno gene has a single Sac I site at the 351st base and VP-1 gene has a single EcoR I site at the 1722nd base. The hatched region corresponds to the locus that is amplified by PCR.

bp, by EcoR I. The same DNA sample as the one used for electrophoresis on lanes a, b, and c in Fig. 3 was digested by EcoR I, and the resultant fragments were analyzed on agarose gels. Two fragments coinciding with $77 \mathrm{bp}$ and $130 \mathrm{bp}$ were detected on lanes $a^{\prime}, b^{\prime}$ and $c^{\prime}$ in Fig. 3. A thin band of $207 \mathrm{bp}$ on lane $\mathrm{a}^{\prime}$ was the undigested residue of PCR products (Mad-1 DNA) by EcoR I. Sac I digests JCV DNA at the 351st base pair and produces $66 \mathrm{bp}$ and $141 \mathrm{bp}$ fragments from PCR products amplified with agno primers (Fig. 1). A result similar to Eco R I/VP-1 was also obtained for Sac I/ agno gene (data not shown). These results mean that EcoR I and Sac I digesting sites of PCR products in patient's DNA were the same sequence as those in JCV DNA of Mad-1, prototype JCV. No amplification of either agno or VP-1 genes occurred in DNA prepared from peripheral lymphocytes of 20 healthy volunteers. Therefore, the present technique of PCR and product analysis are valid for our purposes.

\section{Detection of JCV DNA in clinical cases}

In peripheral lymphocytes, agno gene was detected in a patient of ATL and in a patient of CLL (Fig. 4). Table 1 summarizes the result of all cases tested. Lymphocytes that were positive for agno gene were also positive for VP-1 gene. Agno gene 


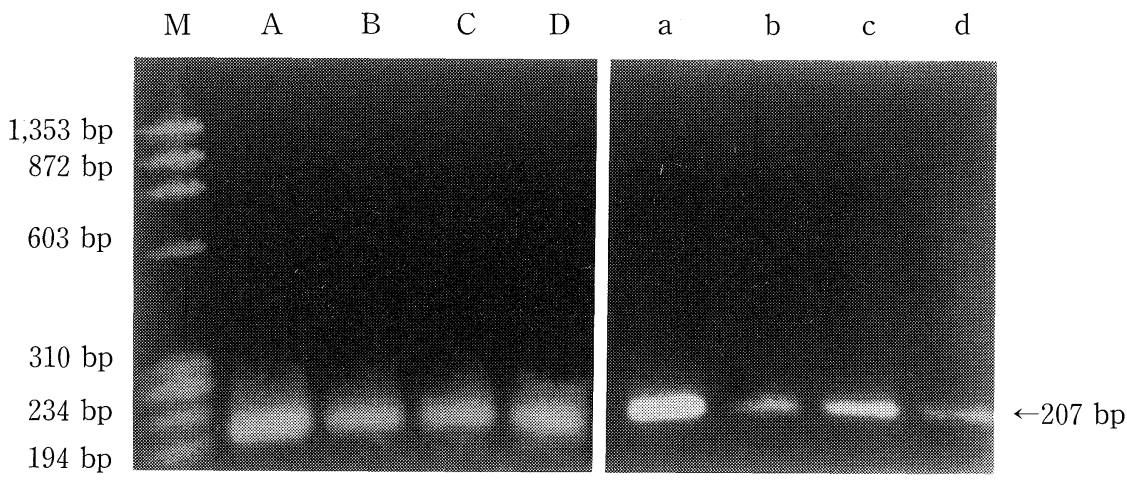

Figure 2. Detection of JCV DNA in peripheral lymphocytes of a PML patient. M: molecular weight marker ( $\phi \times 174-H a e$ III digest), A; a: prototype Mad-1, B; b: lymphocytes before sorting, C; c: B lymphocytes after sorting, D; d: T lymphocytes after sorting, A D: agno primers, a d: VP-1 primers.

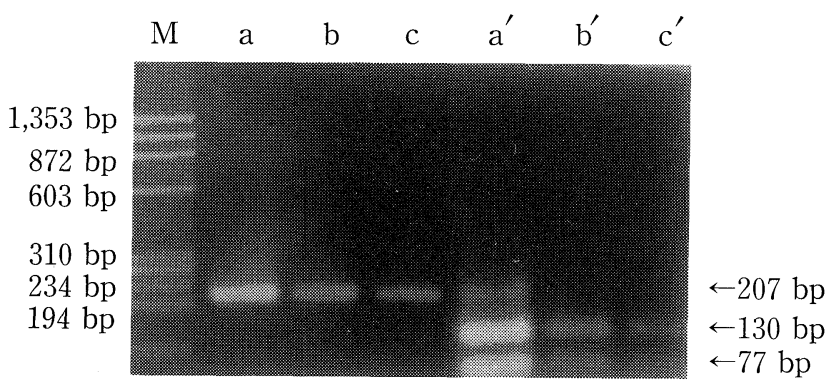

Figure 3. Detection of a part of VP-1 genome in peripheral lymphocytes of patients with hematological malignancy. M: molecular weight marker ( $\phi \times 174-H a e$ III digest), a; $a^{\prime}:$ Mad-1 strain (JCV prototype), b; b': ATL (Adult T cell leukemia), $\mathbf{c}^{\prime} ; \mathbf{c}^{\prime}: \mathbf{A L L}$ (Acute lymphocytic leukemia), $a^{\prime}$; $b^{\prime}$; $c^{\prime}$ : PCR products digested with EcoR I. and VP-1 gene cover about half the JCV genome (Fig. 1). Therefore, the result suggests the presence of the total viral genome, though it does not necessarily mean the presence of the virus particle. The clinical background of 7 patients who have JCV genome -positive lymphocytes is listed in Table 2. Most of these patients showed some degree of hematological malignancy except for one SLE and one ML, containing over $40 \%$ of leukemic cells in peripheral blood. In particular, $98 \%$ of lymphocytes of patient No. 2 (ALL) were blast type, and patient No. 6 (CLL) had $90 \%$ of leukemic cells (CD5 positive). Patient No. 1 (MDS) was diagnosed as leukemia and contained $40 \%$ of leukemic cells. As seen in Table 2, there was no common feature in age and time after diagnosis. In hematological diseases, 5 patients out of 35 tested male cases and one out of 17 female cases contained JCV DNA in their lymphocytes. Only one SLE patient being positive for the presence of JCV genome was

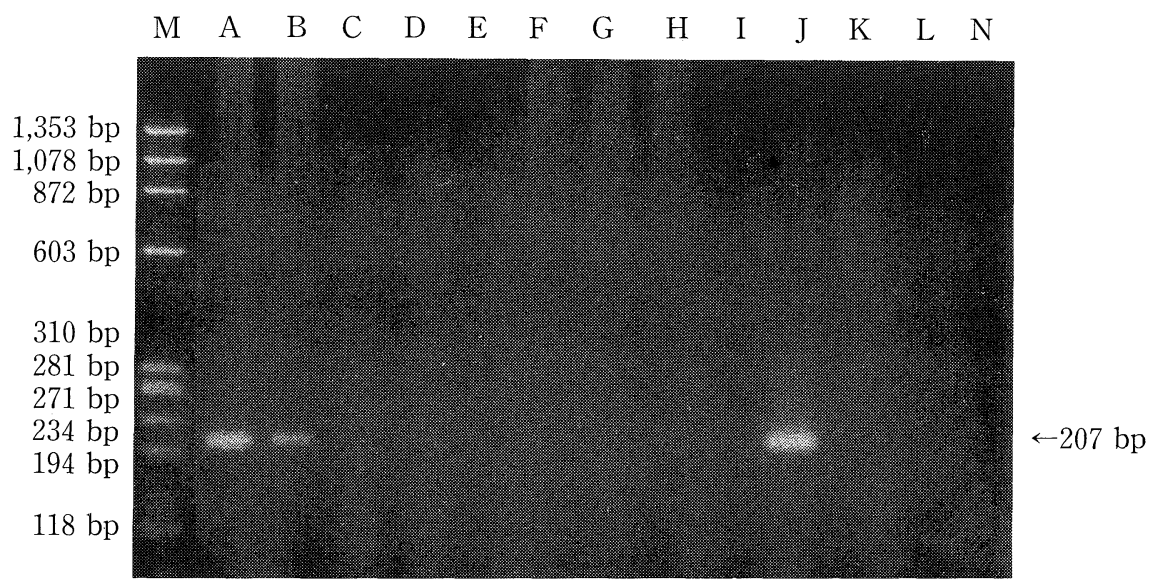

Figure 4. Detection of agno gene in peripheral lymphocytes prepared from 12 patients

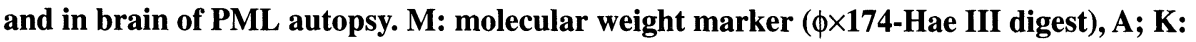
CLL (chronic lymphocytic leukemia), B: ATL (adult T cell leukemia), C; D; E; F; H; L: ML (malignant lymphoma), G: CML (chronic myelogenous leukemia), I: SLE (systemic lupus erythematosus), J: brain of PML autopsy, N: MDS (myelodysplastic syndrome). 
Table 1. Detection of JCV DNA in Peripheral Lymphocytes Prepared from Patients with Hematological Diseases or SLE

\begin{tabular}{lcccc}
\hline \multirow{2}{*}{ Diseases } & & \multicolumn{2}{c}{ Sex } & \\
\cline { 4 - 5 } \multicolumn{1}{c}{ Myelodysplastic syndrome } & Detection of JCV DNA/tested cases & male & female & $(\%)$ \\
\hline Acute lymphoblastic leukemia & $1 / 4$ & 2 & 2 & 25 \\
Adult T cell leukemmia & $1 / 2$ & 0 & 2 & 50 \\
Malignant lymphoma & $2 / 2$ & 2 & 0 & 100 \\
Chronic lymphocytic leukemia & $1 / 28$ & 20 & 8 & 3.6 \\
Acute myelogenous leukemia & $1 / 7$ & 5 & 2 & 14.3 \\
Chronic myelogenous leukemia & $0 / 5$ & 3 & 2 & 0 \\
Multiple myeloma & $0 / 2$ & 1 & 1 & 0 \\
\hline Total & $0 / 2$ & 2 & 0 & 0 \\
\hline Systemic lupus erythematosus & $6 / 52$ & 35 & 17 & 11.5 \\
\hline
\end{tabular}

DNA was extracted from peripheral lymphocytes and analyzed by PCR using agno primers. Cases positive for agno primers were also positive for VP-1 primers.

female, but 17 SLE patients tested were incidentally all female in our cases (Table 1). Although the total number, seven, of positive cases was too small to draw any conclusion, higher leukemic cells content and the sex distinction, male, seem to be the indications with the presence of JCV genome. Reactivation of latent JCV and the transfer from kidney to lymphocytes tend to readily occur in male patients having malignant-type cells in their peripheral blood.

\section{Detection of JCV DNA in B and T lymphocytes}

As shown in Fig. 2, we found JCV DNA in T lymphocytes as well as in B of PML patients. In the PML autopsy specimen, JCV DNA was found not only in brain but in kidney, liver and spleen (data not shown). To test the possibility that the presence of JCV DNA in T lymphocytes is characteristic of a PML case, peripheral lymphocytes of a positive ATL patient were sorted out as described in Materials and Methods. Figure 5 shows that even in an ATL case, JCV DNA exists not only in B lymphocytes but also in T. Once JCV is reactivated, it presumably migrates to $\mathrm{T}$ lymphocytes concomitantly with migration to $\mathrm{B}$ in vivo.

\section{Time course of PCR}

Glial cells of brain and B lymphocytes contain nuclear factors which bind to the regulatory sequence and support the growth of JCV $(6,7)$. JCV is thought to be unable to grow in T lymphocytes without these growth supporting factors. Therefore, the amount of JCV DNA in T lymphocytes is expected to be less than in B. Although we adjusted the amount of template DNA by preparing from an equal number of cells, PCR products did not necessarily reflect the initial quantity of templates after 35 cycles. Starting from 26 cycles, the amount of PCR products with agno primers was surveyed by the intensity of luminescence of ethidium bromide with the advance of the

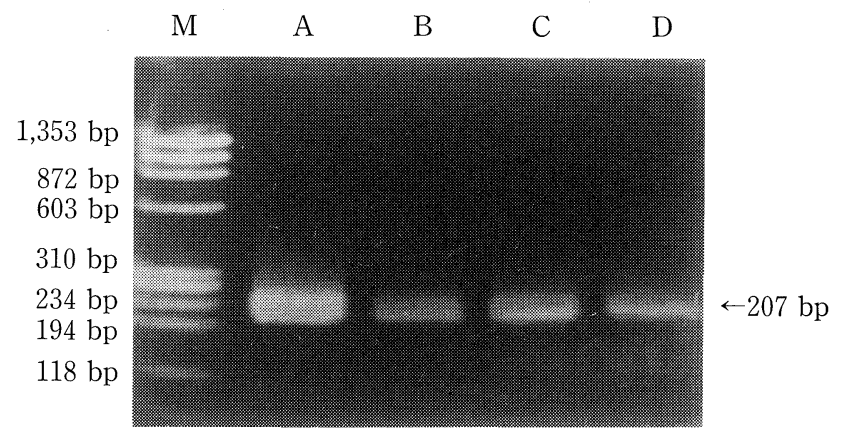

Figure 5. Detection of agno gene in lymphocytes of an ATL patient. A: Mad-1 strain (JCV prototype), B: peripheral lymphocytes before sorting, C: B lymphocytes after sorting, D: T lymphocytes after sorting.

PCR cycle. As seen in Fig. 6, PCR products were barely detected after 26 cycles, but increased exponentially with the increment of PCR cycle. No noticeable difference was observed between $\mathrm{T}$ and B lymphocytes of the ATL patient (lanes C, D in Fig. 6). A similar result was also obtained for the PML patient (data not shown). Despite the presence of growth supportive factors for JCV in B lymphocytes, the amount of JCV genome in B lymphocytes was almost equivalent to $\mathrm{T}$.

\section{Discussion}

The method applied for detection of JCV DNA in this report was tested by comparing the length of PCR product fragments after digestion by restriction enzymes. We previously reported that the base sequences of PCR products completely coincided with those of JCV DNA prepared from Mad-1 strain 
$\begin{array}{lllllllll}M & \text { A } & \text { B } & \text { C } & \text { D } & \text { E } & \text { F } & \text { G }\end{array}$

(1)

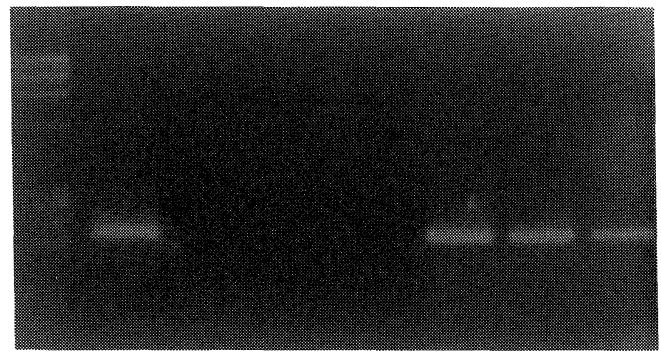

(2)

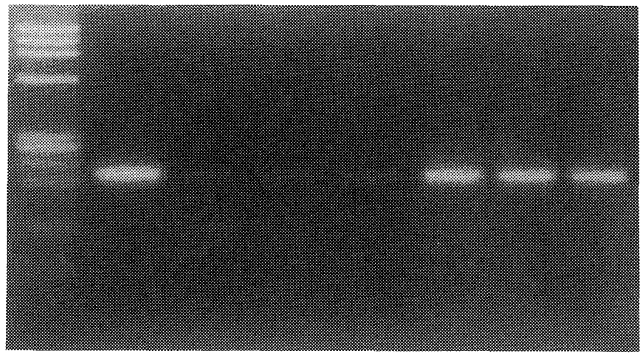

(3)

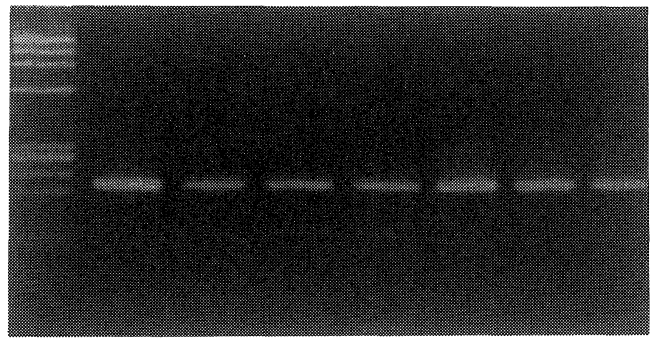

Figure 6. Time course of PCR. (1): 26 cycles, (2): 29 cycles, (3): 35 cycles. M: molecular weight marker $(\phi \times 174-H a e$ III digest), A: 1 ng DNA of Mad-1 strain (JCV prototype), B: peripheral lymphocytes of ATL befor sorting, C: B lymphocytes of ATL after sorting, D: T lymphocytes of ATL after sorting, E: 0.1 ng DNA of Mad-1 strain, F: 0.02 ng DNA of Mad-1 strain, G: 0.01 ng DNA of Mad-1 strain. after TA cloning the PCR products into Bluescript II followed by cycle sequencing (data not shown). All the cases positive for agno gene were also positive for VP-1 gene. As seen in Fig. 1, agno gene and VP-1 gene occupy about half the JCV genome. Therefore the positive results of the current PCR imply the presence of JCV genome in template DNA preparations.

Lymphocytes prepared from seven cases ( 6 cases of hematological diseases and one SLE case) contained JCV DNA that normally exists in the kidney of healthy people under the latent state. These JCV DNA positive patients showed no neurological symptoms of PML as yet. This finding supports the current view that with the progression of immunodeficiency, $\mathrm{JCV}$ in kidney is reactivated and migrates into brain via lymphocytes (3). Since B (not T) lymphocytes contain growth supporting factors of JCV, they are supposed to be the only conveyer of JCV. However, we detected JCV DNA in T lymphocytes as well as B. This was true not only in PML patients whose brain, kidney and liver contained JCV genome, but in all JCV DNA positive cases tested so far. To examine the possibility of contamination of B lymphocytes in $\mathrm{T}$ preparation and vice versa, RT-PCR was run using commercially available HTLV primers. The template DNA was the same DNA fraction as the one used to amplify JCV DNA in ATL (patient No. 3 in Table 2). HTLV genome was detected only in T cell fraction and not at all in B cell fraction by electrophoretic analysis (unpublished observations). In our hands, the sensitivity of detection by RT-PCR was one viral copy per cell and that is comparable to the case of JCV genome. Therefore the purity assessed by a cell sorter was concluded to be sufficient for this study. In established cell lines, cells (Raji, RPMI 8226, RPMI 1788 and KOPN-1) derived from B lymphocytes contained JCV DNA, but not cells (Molt-4 and Jurkat) derived from T lymphocytes (data not shown). In vivo, reactivated JCV probably migrates to $\mathrm{T}$ lymphocytes concomitant with the transfer to B lymphocytes. During the course of establishment of cell lines, JCV in T lymphocytes would be diluted out by cell multiplication and eventually lost from the population of these cell lines.

Table 2. Clinical Information of JCV DNA-positive Patients

\begin{tabular}{cccccc}
\hline $\begin{array}{c}\text { Patient } \\
\text { No. }\end{array}$ & Disease & Age/Sex & $\begin{array}{c}\text { Duration after diagnosis } \\
\text { (years) }\end{array}$ & $\begin{array}{c}\text { Leukemic cells } \\
(\%)\end{array}$ & Treatment \\
\hline 1 & MDS & $69 / \mathrm{M}$ & 0.3 & 40 & none \\
2 & ALL & $68 / \mathrm{F}$ & 0.1 & 98 & none \\
3 & ATL & $54 / \mathrm{M}$ & 1 & 60 & none \\
& (chronic) & & & 70 & etoposide/prednisolone \\
4 & ATL & $47 / \mathrm{M}$ & 3 & 0 & CHOP (6 courses) \\
& (chronic) & & & 90 & CHOP (9 courses) \\
5 & ML & $80 / \mathrm{M}$ & 1.5 & 0 & prednisolone \\
6 & CLL & $59 / \mathrm{M}$ & 2 & 5 & \\
7 & SLE & $36 / \mathrm{F}$ & &
\end{tabular}

MDS: myelodysplastic syndrome, ALL: acute lymphoblastic leukemia, ATL: adult T cell leukemia, ML: malignant lymphoma, CLL: chronic lymphocytic leukemia, SLE: systemic lupus erythematosus, CHOP: cyclophosphamide+doxorubicin+vincristine+prednisolone. 


\section{Distribution of JC Virus DNA in Peripheral Blood}

Figure 6 shows that there is no noticeable difference in the amount of PCR product using template DNA prepared from the same number of $\mathrm{T}$ and $\mathrm{B}$ lymphocytes. We estimated the copy number of JCV genome in these cells on the basis of the following three assumptions; 1) Mad-1 strain (in pJC) consists of $9.3 \times 10^{3} \mathrm{bp}$ in which one molecule of agno gene exists; 2 ) a fragment of $207 \mathrm{bp}$ is amplified with agno primers in PCR; and 3) DNA in a human cell is $3 \times 10^{9} \mathrm{bp}$. Comparing the intensity of luminescence of PCR products with the standard Mad1 template of $0.01 \mathrm{ng}$, the template prepared from lymphocytes was extrapolated into cycle 0 from cycle 26,29 and 35 . One or 2 copies of JCV genome was calculated to be in a B lymphocyte as well as in a T prepared from ATL (No. 3 in Table 2). A similar result was obtained in lymphocytes of PML patients and also in cell lines derived from B lymphocytes. These is no information regarding the state of JCV genome in cells. It possibly stays in the nucleus as a free DNA molecule or a DNA recombined with cellular chromosomes or in the cytoplasm as a DNA in a viral particle. These possibilities may be elucidated by in situ PCR hybridization.

As shown in Table 2, 3 and 5 years passed after diagnosis for patients No. 4 and No. 7, respectively. To date, neither patient has shown symptoms of PML, though JCV DNA already migrated to lymphocytes from kidney. Some unknown barrier in addition to immunodeficiency seems to exist in the host preventing JCV to migrate in brain, and to multiply eventually inducing PML.

The enhancer/promoter region (regulatory sequence) of JCV genome (Fig. 1) isolated from the brain of a PML autopsy case shows a base sequence different from that of the JCV genome isolated from the kidney of healthy subjects $(8,9)$. It has been suggested that this structural alternation is the cause of PML onset (10). In the seven patients in whom JCV DNA was detected in the peripheral blood, we would like to clarify whether the enhancer/promoter region has already undergone the same structual change as the JCV in the PML patients or retained the same structure as the JCV in the kidney or urine of normals. It may be hypothesized that if the enhancer/promoter region does not undergo the structural alteration into a PML type, JCV could not migrate from the kidney to lymphocytes; or, after migrating and arriving at the brain via lymphocytes first, JCV would then alter the structure of the enhancer/promoter region and proliferate there to cause the onset of PML. The elucidation of these points would be a breakthrough in the understanding of the specificity of JCV proliferation and the triggering of PML.

Acknowledgements: We are grateful to Drs. H. Ikeda and Y. Suzuki (Aichi Medical University, Nagakute) for providing autopsy specimens of PML.

\section{References}

1) Major EO, Amemiya K, Tornatore CS, Houff SA, Berger JR. Pathogenesis and molecular biology of progressive multifocal leukoencephalopathy, the JC virus-induced demyelinating disease of the human brain. Clin Microbiol Rev 5: 49-73, 1992.

2) Padgett BL, Walker DL, ZuRhein GM, Eckroade RJ, Dessel BH. Cultivation of papova-like virus from human brain with progressive multifocal leukoencephalopathy. Lancet 1: 1257-1260, 1971.

3) Nagashima K. Progressive multifocal leukoencephalopathy: Clinical and pathological study. in: Infection of Slow Viruses and Prion. Yamanouchi K, Tateishi J, Eds. Kindai Shuppan, Tokyo, 1995: 109-123 (in Japanese).

4) Imamura A. A study of diagnostic procedures for JC viral infection by polymerase chain reaction (PCR) method with newly established primer. J Aichi Med Univ Assoc 20: 95-109, 1992 (in Japanese, Abstract in English).

5) Frisque RJ, Bream GL, Cannella MT. Human polyomavirus JC virus genome. J Virol 51: 458-469, 1984.

6) Major EO, Amemiya K, Elder G, Houff SA. Glial cells of the human developing brain and $\mathrm{B}$ cells of the immune system share a common DNA binding factor for recongnition of the regulatory sequences of the human polyomavirus, JCV. J Neurosci Res 27: 461-471,1990.

7) Rieckmann P, Michel U, Kehrl JH. Regulation of JC virus expression in B lymphocytes. J Virol 68: 217-222, 1994.

8) Loeber G, Dorries K. DNA rearrangements in organ-specific variants of polyomavirus JC strain GS. J Virol 62: 1730-1735, 1988.

9) Dorries K. Progressive multifocal leukoencephalopathy: analysis of JC virus DNA from brain and kidney tissue. Virus Res 1: 25-38, 1984.

10) Yogo $Y$, Kitamura $T$, Sugimoto $C$, et al. Isolation of a Possible archetypal JC virus DNA sequence from nonimmunocompromised individuals. $J$ Virol 64: 3139-3143, 1990. 